\title{
Evaluation of Mechanical Properties of Skeletal Muscle with Strain Injury*
}

\author{
Daisuke ITO $^{* *}$, Sota YAMAMOTO***, Eiichi TANAKA ${ }^{\dagger}$, Koji MIZUNO $^{\dagger}$, \\ Masahito HITOSUGI ${ }^{\dagger \dagger}$ and Shogo TOKUDOME ${ }^{\dagger \dagger}$ \\ ** Japan Automobile Research Institute \\ 2530, Karima, Tsukuba, Ibaraki, 305-0822, Japan \\ *** Department of Engineering Science and Mechanics, Shibaura Institute of Technology \\ 3-7-5, Toyosu, Koto-ku, Tokyo, 135-8548, Japan \\ $\dagger$ Department of Mechanical Science and Engineering, Nagoya University \\ Furo-cho, Chikusa-ku, Nagoya, Aichi, 464-8603, Japan \\ $\dagger$ Department of Legal Medicine, Dokkyo Medical University \\ 880 Kitakobayashi, Mibu-machi, Shimotsuga-gun, Tochigi, 321-0293, Japan \\ E-mail: dito@jari.or.jp
}

\begin{abstract}
To discuss the mechanism of muscle strain injury from biomechanical viewpoints, the change of mechanical properties of injured skeletal muscle was evaluated experimentally. We induced strain injury to rabbit tibialis anterior muscle by applying a single tension loading/unloading process under the conditions of various stretch amplitude, stretch rate, and muscle activation in situ. The results showed that isometric contractile force decreased with increasing stretch amplitude during the injury-induction process. Multiple regression analyses were conducted for the results and they showed that the stretch amplitude played a dominant role in the decrease of the contractility. The failure stress decreased significantly with the increasing stretch amplitude and dissipation energy during the injury-induction process. Moreover, the failure stresses of the muscle injured under unstimulated conditions obviously decreased in comparison with those of the muscles in intact and injured under stimulated conditions. On the basis of these results and previous experimental studies, we concluded that muscle activity played a significant roll to prevent the decrease of the failure stress and that the damage mechanism of strain injury depended on the muscle activity during injury-induction process.
\end{abstract}

Key words : Skeletal Muscle, Mechanical Properties, Strain Injury, Experimental Approach, Muscle Activity, Failure Stress

\section{Introduction}

Skeletal muscle has received a lot of attention in recent years in the field of impact biomechanics. For example, several experimental studies and simulations for car crash safety showed that the muscle contractile force affects the overall kinematics of the human body during whole impact events, occurrence of occupant injuries, and injury severities ${ }^{(1)-(3)}$. In these accidents, muscle is also subjected to rapid extension and direct impact, which induce muscle injury. Muscle injury causes functional disabilities on body motion and takes long periods to recover. In cases of severe injury, the period for recovery is comparable with that of bone fracture. Therefore, it is very important to elucidate the mechanical and damage properties of skeletal muscle from the viewpoint of injury prevention.

Strain injury is one of the most common muscle injuries among athletes, for example, hamstring injuries in football ${ }^{(4)}$ and tennis $\operatorname{leg}^{(5)}$. It is also observed in traffic injury such as whiplash injury ${ }^{(6)}$. Strain injury is caused primarily by repeated stretching or acute single stretching in the direction of the muscle fiber. When a muscle is exposed to a loading/unloading stretching, the muscle contractile force decreases significantly ${ }^{(7)}{ }^{(13)}$, and his-

*Received 3 Oct., 2011 (No. 11-0593) [DOI: 10.1299/jbse.7.156]

Copyright (c) 2012 by JSME 
tological damage ${ }^{(7),(10),(12),(13)}$ and damage at electron-microscopic level ${ }^{(9)}$ can be observed. Many researchers have investigated the effects of the mechanical factors, such as stretch amplitude, stretch rate and muscle activity on the decrease of the contractile force, and have discussed the relation between the decrease and microstructural damage.

While a lot of attention have been paid for the decrease of contractile force, change of failure properties for strain injury have not been discussed enough. Some studies showed that the muscle activity during injury-induction process does not affect the failure properties of the injured muscle ${ }^{(8),(10)}$. Other researchers, on the other hand, reported that muscle contraction affects connective tissue damage ${ }^{(12)}$ and fracture mode of muscle at electron-microscopic level (14). The effects of stretch rate on damage are also still unclear because only a stretching at a single constant rate was applied to muscle for strain injury-induction in most of literatures.

It is expected that evaluation of failure properties enable us not only to quantify macroscopic failure properties of injured muscle but also to estimate mechanical and damage properties of passive element of muscle. Concerning this subject, it was reported in previous studies $^{(15),(16)}$ that there is no significant difference between the failure force under the maximum activated condition and that under passively-stretched condition, and that the contractile force near the breaking point of muscle is relatively small. Hence, it can be thought that the failure properties of muscle reflect the mechanical properties of passive elements of muscle, such as epimysium, perimysium, endomysium, because the muscle generates little active force around the breaking point. However, the effects of stretch amplitude, stretch rate, and muscle activity during injury-induction on failure properties are still unclear, and thus it can be said that systematic quantitative evaluation of failure properties of damaged muscle is required.

The purpose of this study is to evaluate the effects of muscle strain injury on failure properties and contractile properties. For this purpose, we examine the strain injury of rabbit tibialis anterior (TA) muscle induced by a single stretching under various stretch amplitude, stretch rate, and muscle activation conditions in situ. The effects of strain injury on the biomechanical factors, such as changes of contractile force, the failure stresses, and failure stretch ratios were investigated by using multiple regression analyses.

\section{Methods}

\subsection{Animals and animal care}

We used TA muscle of female Japanese white rabbit. Fifty-seven rabbits (body mass $2.82 \pm 0.22 \mathrm{~kg}$, mean \pm S.D. $)$ were used for strain injury tests and seven of them $(2.88 \pm$ $0.21 \mathrm{~kg}$ ) were used as the control. The procedure of animal experiments in the following was approved by Nagoya University Animal Experiment Committee in accordance with the Nagoya University Regulations on the Treatment of Animal Experiments.

\subsection{Surgical procedures}

The following procsedure was used for the preparation. The animals were anesthetized by pentobarbital sodium $(30 \mathrm{mg} / \mathrm{kg})$ or isoflurane $(2.0 \% \mathrm{vol})$. Then, the TA muscle and deep peroneal nerve were exposed. Two dot markers were stained on the surface of the proximal and distal ends of the muscle belly by nigrosine to determine the muscle length. The initial length of the muscle was defined as the in situ distance between the dots at a 90-degree angle to the ankle joint. Next we inserted a Kirschner's wire $(\phi=7 \mathrm{~mm})$ into the tibial condyle to hold the tibia at a jig. A cross-sectional area, which was used for calculation of nominal stress, was measured by an area micrometer, which determines the cross-sectional area by compressing the specimens to rectangular sections of known width and measuring the heights of the sections by means of a micrometer head ${ }^{(17),(18)}$. The cross-sectional area was measured at a point one-third of muscle's length from the distal end. Finally, the distal tendon of the TA muscle was cut and directly gripped by a tooth-like jig. 


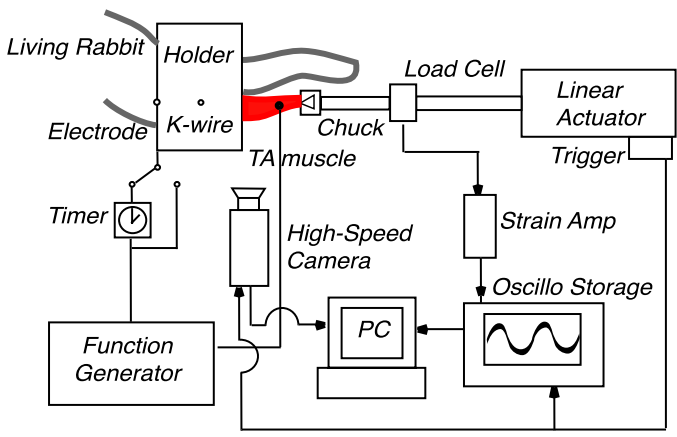

Fig. 1 Schematic diagram of experimental apparatus.

\subsection{Experimental Apparatus}

Mechanical experiments were conducted using an experimental apparatus developed for muscle strain injury tests shown in Fig.1. This experimental apparatus consists of a motorized linear stage with a linear motor type actuator (GLM-20, THK), a knee holder, a load cell (LUR-A-2KNSA1, Kyowa), a digital high-speed camera (MEMRECAM fxK3, NAC Image Technology), a digital oscilloscope (DS-4264ML, IWATSU), a PC and a function generator (SG-4105, IWATSU). An animal is put in lateral recumbent position after the surgical preparation described above. The wire inserted in the tibial condyle is fixed on the knee holder, and the jig gripping the distal tendon is fixed at the linear stage. The load cell is placed at the jig gripping the distal tendon and detects the load on the muscle directly. Signals of the load cell are instantaneously stored on the digital oscilloscope during experiments, and then transmitted to a PC. The high-speed camera is set up in front of the anterior surface of TA muscle and records the movements of the markers. This camera is started by a trigger signal from the motorized linear stage to be synchronized with the output of the load cell. The movie file is transmitted from the memory of the camera to the PC after each loading procedure. The stretch amplitude of the muscle was calculated by measuring the distance between the markers on the surface of the muscle. To evaluate the effects of stretch rate on strain injury and mechanical properties of muscle, a change of extension rate during experiments should be stepwise to minimize acceleration and deceleration phases. Thus we use a linear motor type actuator that can operate with high acceleration and deceleration, and with high accuracy of positioning. The function generator is used to stimulate the muscle. A stimulation signal is transmitted to the muscle by a pair of thin wire electrodes for EMG measurement (ST. Steel 7 Strand, Teflon, A-M Systems), one of which is inserted into the distal end of the muscle belly and the other is directly clipped onto the deep peroneal nerve.

\subsection{Mechanical Test Procedure}

Firstly, the muscle was cyclically stretched with $7 \mathrm{~mm}$ amplitude (less than stretch ratio of 0.13 ) at the rate of $7 \mathrm{~mm} / \mathrm{s}$ as a preconditioning referring to Taylor et al. ${ }^{(19)} \mathrm{Next}$, we performed an isometric contraction test to evaluate the initial contraction force of the specimen. The specimen was stimulated by pulse signal with a frequency of $50 \mathrm{~Hz}$ during 10 seconds. The voltage of the amplitude of the pulse for maximum contraction was determined as tenfold the threshold for twitching following Scott et al. ${ }^{(20)}$

After enough interval time to recover from fatigue caused by the first isometric contraction test (longer than 5 minutes), we induced a strain injury by applying a tension loading/unloading at 2, 20, $200 \mathrm{~mm} / \mathrm{s}$ to the muscle under maximally stimulated condition (stimulated group) or without stimulation (unstimulated group). The conditions of stretch amplitude were set at $0.2,0.25$ or 0.3 of the in situ length referring to the results of Hasselman et al. ${ }^{(10)}$ Dissipation energy was defined as the area of the region enclosed by the nominal stress-stretch ratio curve during this injury-induction process. As mechanical evaluations of muscle strain injury, we conducted an isometric contraction test again, and finally performed a tensile test 
of the injured muscle at $200 \mathrm{~mm} / \mathrm{s}$ extension rate under the maximally stimulated condition. In the control group, the muscle was stretched until breaking without the injury-induction process, and in this case we gave the same interval times between each mechanical evaluation procedure as those in the injury induced group. Figure 2 shows the schematic diagram of the test procedure.

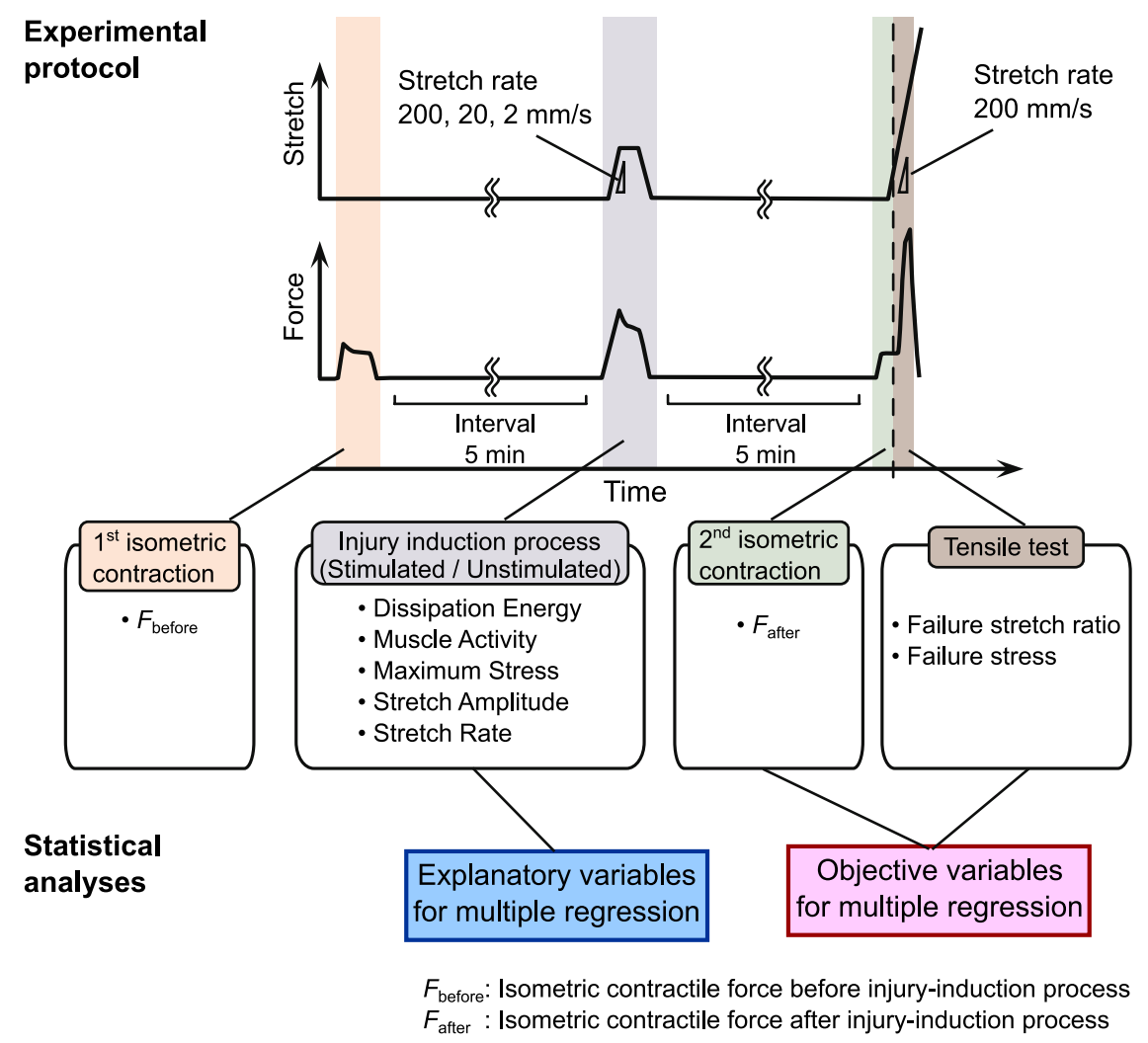

Fig. 2 Schematic diagram of test procedure.

\subsection{Statistical analyses}

In order to find out the biomechanical factors that strongly influence the contractility and failure properties, we performed multiple regression analyses. Before performing the regression analyses, the data were checked for multicollinearity by using the correlation matrix and the variance inflation factor (VIF). We selected the ratio $R_{\text {iso }}\left(=F_{\text {after }} / F_{\text {before }}\right)$ of the isometric contractile force $F_{\text {after }}$ after injury-induction process to the force $F_{\text {before }}$ before the process, failure stretch ratio, and failure stress as the objective variables, and stretch rate, muscle activity, stretch amplitude, dissipation energy, and maximum stress during injury-induction process as the explanatory variables. We dealt with the muscle activity as category data and assigned number 1 to the stimulated group and number 0 to the unstimulated group. The backward selection method was performed, and the threshold of $P$ value for variable removal was set at 0.05 .

Compatibility of multiple regression equation was evaluated by using the coefficient of determination adjusted for the degrees of freedom $R_{\text {adj }}^{2}$, defined by

$$
R_{\text {adj }}^{2}=1-\frac{n-1}{n-p-1}\left(1-R^{2}\right),
$$

where $n$ is the sample size, $p$ is the number of the explanatory variables, and $R$ is the coefficient of determination. The analyses were performed using the statistical programming language "R"(21). 


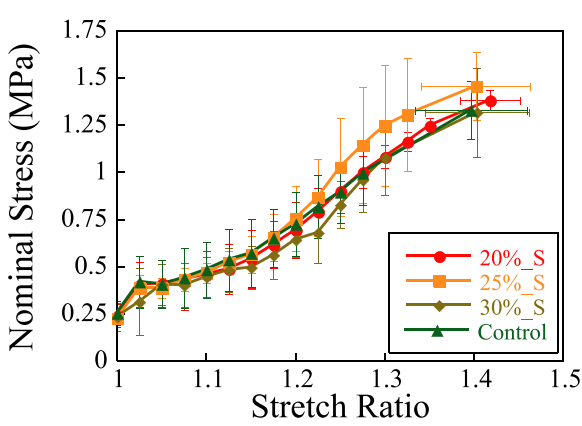

(a) $200 \mathrm{~mm} / \mathrm{s}$ Stimulated group

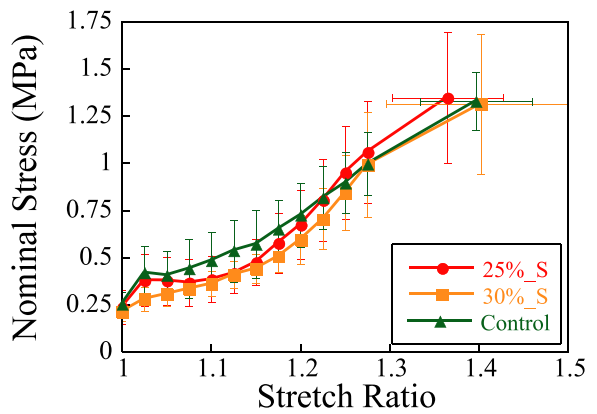

(c) $20 \mathrm{~mm} / \mathrm{s}$ Stimulated group

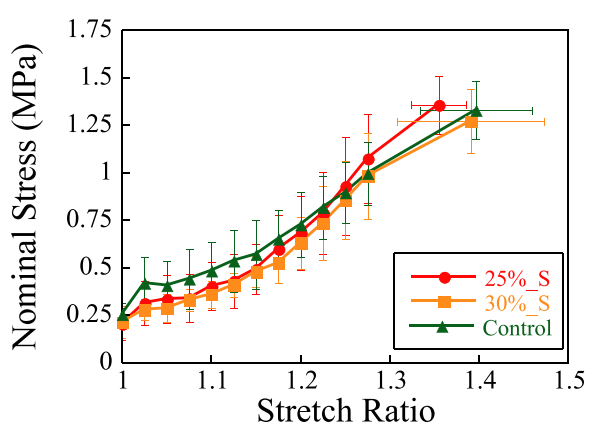

(e) $2 \mathrm{~mm} / \mathrm{s}$ Stimulated group

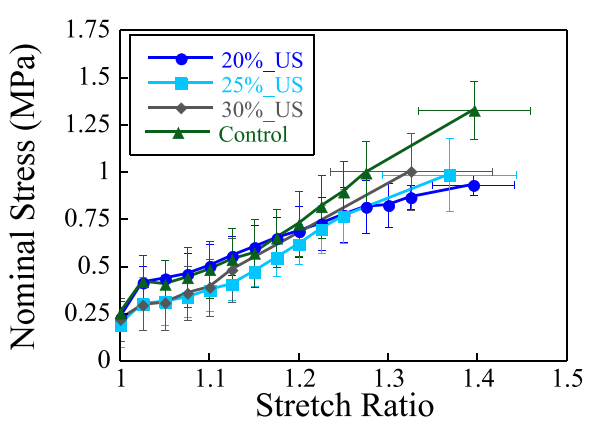

(b) $200 \mathrm{~mm} / \mathrm{s}$ Unstimulated group

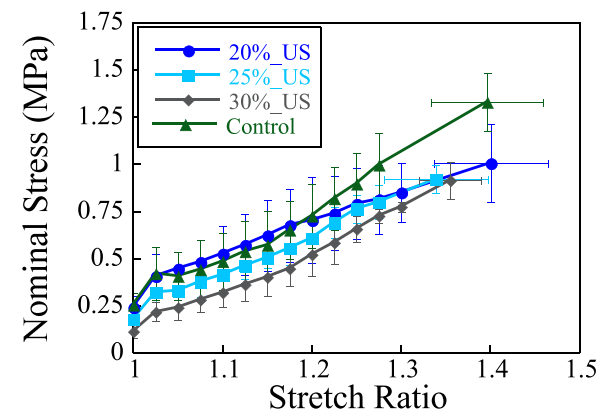

(d) $20 \mathrm{~mm} / \mathrm{s}$ Unstimulated group

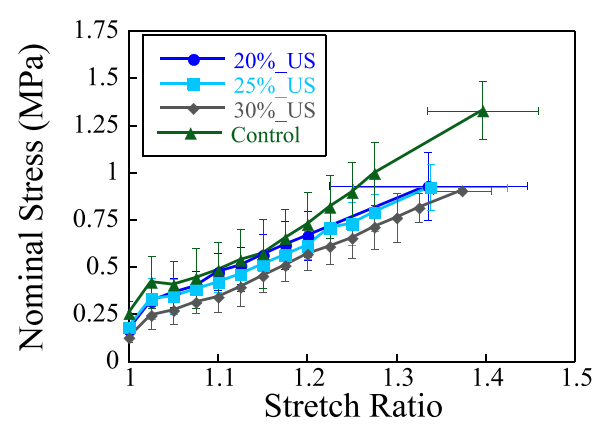

(f) $2 \mathrm{~mm} / \mathrm{s}$ Unstimulated group

Fig. 3 Nominal stress-stretch ratio relations of damaged muscle and undamaged muscle.

("S": stimulated condition, "US": unstimulated condition)

\section{Results}

\subsection{Nominal stress-stretch ratio relations for damaged and undamaged muscle}

Nominal stress-stretch ratio curves of tensile tests for the injured groups and the control group are shown in Fig.3. In these figures, green plots represent the average of the results for the control group and error bars represent the \pm 1 standard deviation (S.D.). In the legends of Fig.3, the words "20 \%", "25\%", and "30\%" show the average results of the muscles injured by stretch amplitudes of 0.2, 0.25 and 0.3 , and abbreviations "S" and "US" added to them mean stimulated and unstimulated conditions, respectively. While the tensile test results for stimulated group (Fig.3(a),(c),(e)) showed no difference against the control group, the failure stresses of the stress-stretch ratio curves for the unstimulated group (Fig.3(b),(d),(f)) decreased and the curves passed below the curve of the control group in all the stretch-rate and stretch-amplitude conditions during injury-induction process.

\subsection{Multiple regression analyses}

In order to examine the influence of each biomechanical factor during injury-induction 
Table 1 Correlation matrix and VIF for multicollinearity

\begin{tabular}{l|ccccc|c}
\hline & \multicolumn{5}{|c|}{ Correlation matrix } & \multirow{2}{*}{$\begin{array}{l}\text { VIF } \\
\end{array}$} \\
$\begin{array}{c}\text { Stretch } \\
\text { Rate }\end{array}$ & $\begin{array}{c}\text { Muscle } \\
\text { Activity }\end{array}$ & $\begin{array}{c}\text { Stretch } \\
\text { Amplitude }\end{array}$ & $\begin{array}{c}\text { Maximum } \\
\text { Stress }\end{array}$ & $\begin{array}{c}\text { Dissipation } \\
\text { Energy }\end{array}$ & \\
\hline Stretch Rate & 1.0000 & & & & & 1.4940 \\
Muscle Activity & -0.1293 & 1.0000 & & & & 1.9231 \\
Stretch Amplitude & -0.1319 & 0.4143 & 1.0000 & & & 2.0351 \\
Maximum Stress & 0.05339 & 0.6608 & 0.4687 & 1.0000 & & 2.8903 \\
Dissipation Energy & 0.2837 & 0.5262 & 0.6306 & 0.7375 & 1.0000 & 3.9382 \\
\hline
\end{tabular}

on the contractility and failure properties, multiple regression analyses were performed. Our analyses began with an examination of potential multicollinearity. Intercorrelations among the explanatory variables and VIF are presented in Table 1. As can be seen from Table 1, most correlation coefficients were less than 0.7 , and all of the VIF for the explanatory variables were less than 10 . Thus, we conclude that we can proceed with our model without undue concern for multicollinearity.

Figures 4, 5, and 6 show the relations between the objective variables and the explanatory ones. The ratio $R_{\text {iso }}$ (Fig. 4), failure stretch ratio (Fig. 5), and failure stress (Fig. 6) are plotted against the stretch amplitude calculated by video analyses, dissipation energy or maximum stress. The closed symbols in the figures express the results of stimulated group and the open symbols express those of unstimulated group. In addition, gray regions in these figures represent the average \pm S.D. of the control group.

Tables 2, 3, and 4 show the results of multiple regression analyses for the relations between the objective variables and the explanatory ones. The short form N.S. (not significant) in each row on the far right means that those objective variables were removed by the backward selection method.

Firstly, we evaluated the ratio $R_{\text {iso }}$, and compared it with the corresponding ratio in the control group. As shown in Fig.4, the isometric contractile force after injury-induction process decreased with the increasing stretch amplitude and became lower than that of the control group. The partial regression coefficient for the stretch amplitude in Table 2 also means that the increase of the stretch amplitude decreases the isometric contractile force. The partial regression coefficient for the stretch rate in Table 2 means that the contractility decreased with the stretch rate.

Next, the failure stretch ratios of all the experimental groups were not different from those of the control group significantly as shown in Fig.5. Although the muscle activity and maximum stress during injury-induction were significantly correlated with the failure stretch ratio as shown in Table 3, these factors may have little effects on failure stretch ratio because of the low compatibility of regression equation derived from the low coefficient of determination $\left(R_{\text {adj }}^{2}=0.1216\right)$.

Finally, as shown in Fig.6, the failure stresses of the unstimulated group are obviously lower than those of the control and stimulated group independently of the stretch amplitude and stretch rate during injury-induction. From the results of multiple regression analyses shown in Table 4, the value of $R_{\text {adj }}^{2}=0.7659$ was the highest of the three regression equations. The high value of $R_{\text {adj }}^{2}$ indicated that the explanatory variable explained $77 \%$ of the variance of the objective variable. Since the partial regression coefficients for the dissipation energy and stretch amplitude were negative, it can be thought that the failure stress decreases with the increase of these mechanical factors. The partial regression coefficients for the muscle activity and maximum stress, on the other hand, were positive and thus it is considered that these variables worked as the factor that inhibited the decrease of failure stress.

\section{Discussion}

4.1. Effects of stretch amplitude, dissipation energy, and stretch rate during injuryinduction on muscle damage

In the results of this study, the contractile force decreased with the increasing stretch 

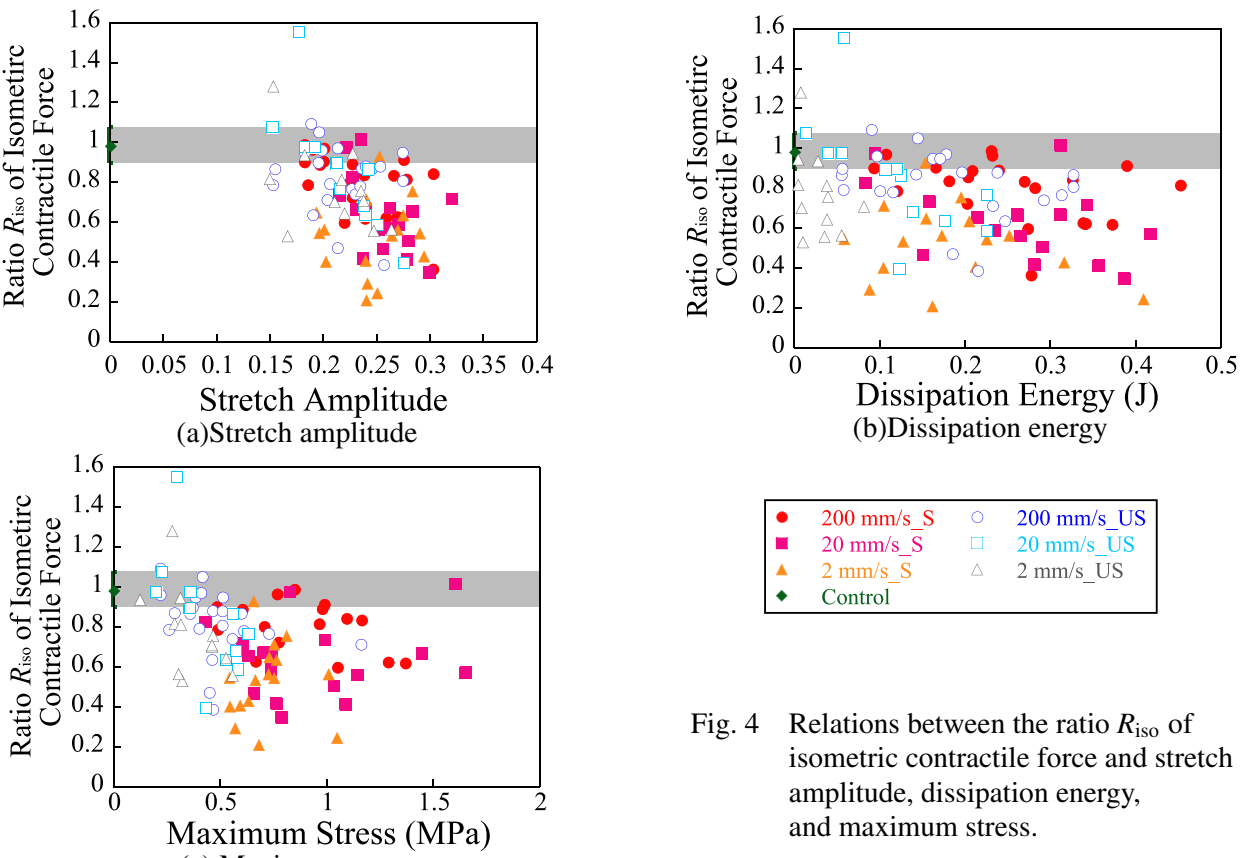

\begin{tabular}{|llll|}
\hline & $200 \mathrm{~mm} / \mathrm{s}_{-} \mathrm{S}$ & $\circ$ & $200 \mathrm{~mm} / \mathrm{s}_{\text {_US }}$ \\
& $20 \mathrm{~mm} / \mathrm{s}_{\mathrm{S}}$ & $\square$ & $20 \mathrm{~mm} / \mathrm{s}_{\text {_US }}$ \\
$\Delta$ & $2 \mathrm{~mm} / \mathrm{s}_{\mathrm{S}}$ & $\triangle$ & $2 \mathrm{~mm} / \mathrm{s}_{-}$US \\
Control & & \\
\hline
\end{tabular}

Fig. 4 Relations between the ratio $R_{\text {iso }}$ of isometric contractile force and stretch amplitude, dissipation energy, and maximum stress.

Table 2 Results of multiple regression analyses for ratio $R_{\text {iso }}$ of isometric contractile force

\begin{tabular}{|c|c|c|c|}
\hline Variables & $\begin{array}{c}\text { partial } \\
\text { regression coefficient }\end{array}$ & $\begin{array}{l}\text { standard partial } \\
\text { regression coefficient }\end{array}$ & $P$-value \\
\hline Dissipation Energy & - & - & N.S. \\
\hline Muscle Activity & - & - & N.S. \\
\hline Maximum Stress & - & - & N.S. \\
\hline Stretch Amplitude & -2.699 & -0.4599 & $<0.001$ \\
\hline Stretch Rate & $5.727 \times 10^{-4}$ & 0.2430 & $<0.001$ \\
\hline \multicolumn{2}{|c|}{$R^{2}=0.2964$} & $R_{\text {adj }}^{2}=0.282$ & \\
\hline
\end{tabular}

amplitude during injury-induction. It supports the previous report of Hasselman et al. ${ }^{(10)}$, in which they pointed out the following relation between stretch amplitude and decrease of isometric contractile force for strain injury. They show that significant decreases of the isometric contractile force are observed when the stretch amplitude at the injury-induction process is larger than 0.23 and the contractile force decreases with the increasing stretch amplitude. Our results showed similar stretch amplitude dependence and demonstrated a high correlation between the decrease of the contractile force and the stretch amplitude during the injuryinduction.

The results of the multiple regression analyses showed that the stretch rate was significant for the severity of strain injury shown in Table 2. In Fig.4, larger decreases of the ratio $R_{\text {iso }}$ under $2 \mathrm{~mm} / \mathrm{s}$ condition were observed in comparison with those under 20 and $200 \mathrm{~mm} / \mathrm{s}$ conditions. Some researchers reported different results for dependence of contractibility on stretch rate. Brooks and Faulkner ${ }^{(11)}$ and Talbot and Morgan ${ }^{(23)}$ reported that rate effects are relatively weak or not significant. These results indicate that the stretch rate has little effects on the decrease of the contractile force. However, the difference of range of stretch rate, experimental protocol, and muscle fatigue, may lead to different dependency on stretch rate, and thus this point is one of the agenda to be examined in the future.

Failure stretch ratio did not change regardless of whether or not loading/unloading process was applied to the muscle. According to Hasselman et al. ${ }^{(10)}$, failure stretch ratio of muscle with strain injury does not decrease in comparison with that of undamaged muscle 

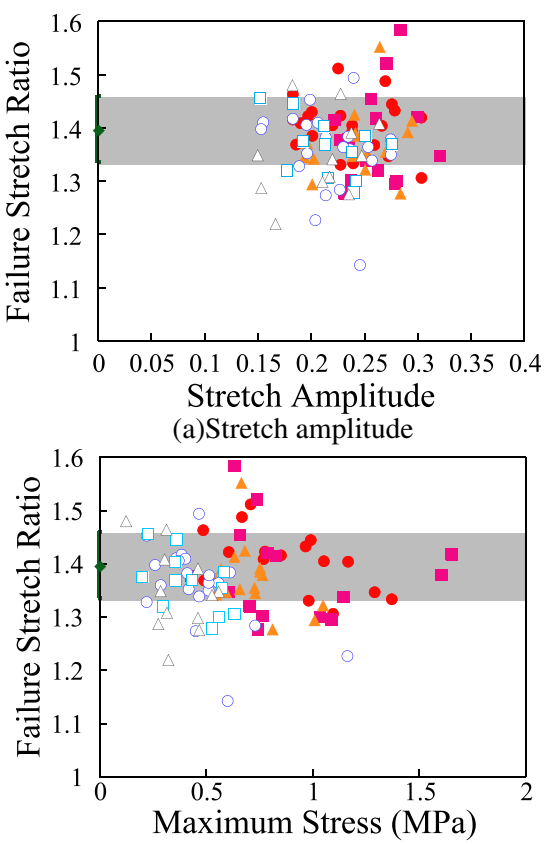

(c) Maximum stress

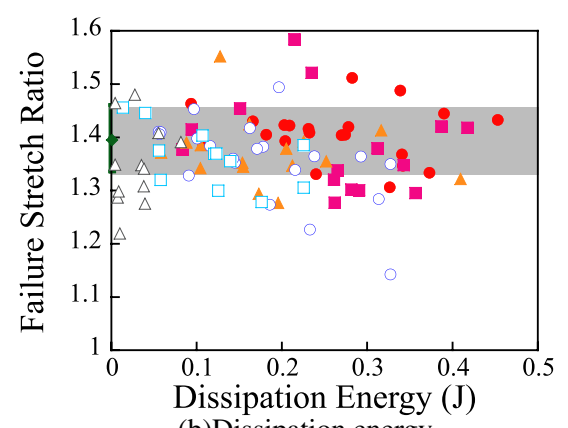

(b)Dissipation energy

\begin{tabular}{|c|c|c|c|}
\hline - & $200 \mathrm{~mm} / \mathrm{s} \_\mathrm{S}$ & 0 & $200 \mathrm{~mm} / \mathrm{s}$ _US \\
\hline - & $20 \mathrm{~mm} / \mathrm{s} \overline{\mathrm{S}}$ & $\square$ & $20 \mathrm{~mm} / \mathrm{s}$ US \\
\hline$\Delta$ & $2 \mathrm{~mm} / \mathrm{s} \overline{\mathrm{S}}$ & $\Delta$ & $2 \mathrm{~mm} / \mathrm{s} \overline{\mathrm{U}} \mathrm{S}$ \\
\hline • & Control & & \\
\hline
\end{tabular}

Fig. 5 Relations between failure stretch ratio and stretch amplitude, dissipation energy, and maximum stress.

Table 3 Results of multiple regression analyses for failure stretch ratio

\begin{tabular}{|c|c|c|c|}
\hline Variables & $\begin{array}{c}\text { partial } \\
\text { regression coefficient }\end{array}$ & $\begin{array}{l}\text { standard partial } \\
\text { regression coefficient }\end{array}$ & $P$-value \\
\hline Dissipation Energy & - & - & N.S. \\
\hline Muscle Activity & 0.06934 & 0.4949 & $<0.001$ \\
\hline Maximum Stress & -0.09696 & -0.4347 & 0.002 \\
\hline Stretch Amplitude & - & - & N.S. \\
\hline Stretch Rate & - & - & N.S. \\
\hline \multicolumn{2}{|c|}{$R^{2}=0.1409$} & $R_{\mathrm{adj}}^{2}=0.1216$ & \\
\hline
\end{tabular}

except for the case in which $90 \%$ of the failure force is applied to TA muscle. Since these results suggest that loading conditions during injury-induction process had little effects on the failure stretch ratio.

From the regression analyses for failure stress, shown in Table 4, the partial regression coefficients for the dissipation energy and stretch amplitude were negative. As already mentioned in Introduction, since the failure properties correspond to the damage of passive elements of muscle, it can be thought that irreversible failure of the passive part of muscle fiber and local rupture of the surrounding connective tissue were induced by excessive loading.

\subsection{Effects of muscle activity during injury-induction on muscle damage}

While the ratio $R_{\text {iso }}$ was independent of the muscle activity during injury-induction process in this study, various results have been obtained in the literature. Uchiyama et al. ${ }^{(12)}$ showed that the contractile force of plantaris muscle stretched under unstimulated condition decreases in comparison with that under stimulated condition, and the unstimulated group requires longer period to recover from strain injury than for the stimulated group. Brooks et al. ${ }^{(9)}$ also reported that significantly larger decreases of isometric contractile force were observed for unstimulated muscles than for stimulated muscles. By contrast, Lieber et al. ${ }^{(22)}$ showed that decrease of the contractile force for stimulated group after repeated eccentric contractions is greater than that for unstimulated group.

As interpretation of these, we deduce that there exists different injury mechanism in the stimulated and unstimulated groups. Although the effects of each factor, such as structural damage of actomyosin, sarcomere disruption, functional loss of excitation-contraction 

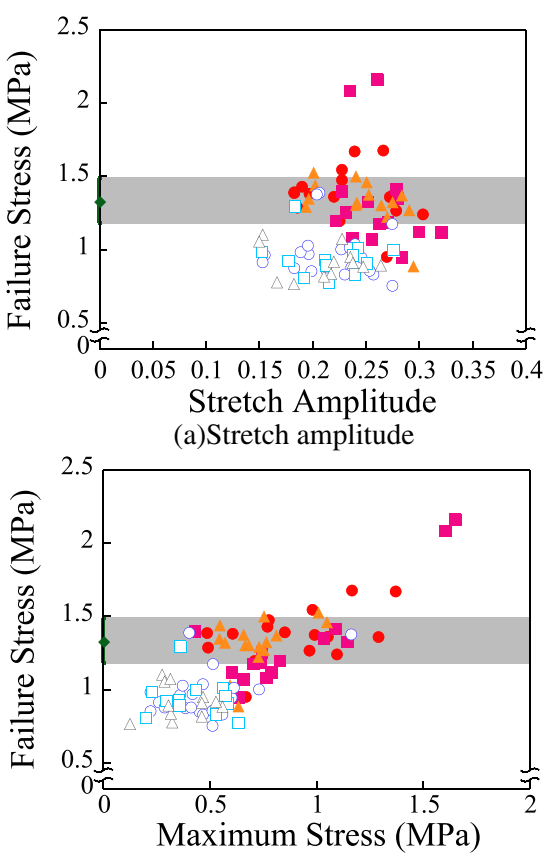

(c) Maximum stress

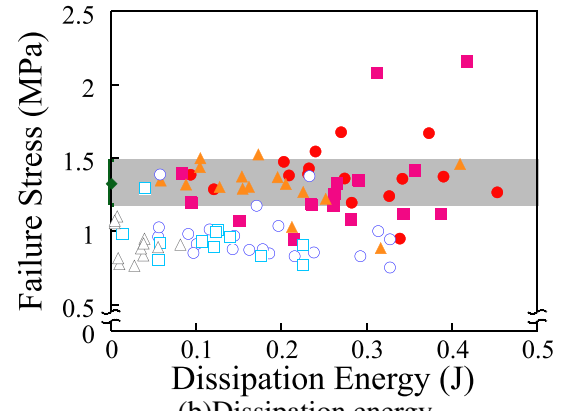

(b)Dissipation energy

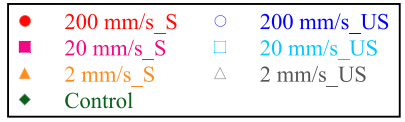

Fig. 6 Relations between failure stress and stretch amplitude, dissipation energy and, maximum stress.

Table 4 Results of multiple regression analyses for failure stress

\begin{tabular}{|c|c|c|c|}
\hline Variables & $\begin{array}{c}\text { partial } \\
\text { regression coefficient }\end{array}$ & $\begin{array}{l}\text { standard partial } \\
\text { regression coefficient }\end{array}$ & $P$-value \\
\hline Dissipation Energy & -0.7533 & -0.3016 & $<0.001$ \\
\hline Muscle Activity & 0.2196 & 0.3968 & $<0.001$ \\
\hline Maximum Stress & 0.7574 & 0.8597 & $<0.001$ \\
\hline Stretch Amplitude & -1.470 & -0.2012 & 0.003 \\
\hline Stretch Rate & - & - & N.S. \\
\hline \multicolumn{2}{|c|}{$R^{2}=0.7762$} & \multicolumn{2}{|l|}{$R_{\mathrm{adj}}^{2}=0.7659$} \\
\hline
\end{tabular}

coupling due to damage of membrane, and functional disability of force transmission by extracellular connective tissue, are different between two groups, the accumulation of the effects of these factors may result in the same magnitude of decrease of contractile force as shown in our study. Several studies have shown that the damage of these factors varies depending on muscle activity during injury-induction process. Connective tissue damage becomes severer when strain injury is induced under unstimulated condition ${ }^{(12)}$. In contrast, a shift of the optimum length in the contractile force-length relation, which is part of contractile properties, occurs with only eccentric contractions ${ }^{(24)-(26)}$. These facts suggest that the contractile force decreases by different causes in the stimulated and unstimulated muscles but consequently exhibit the same magnitude of the macroscopic decrease in our experiments. The variety of results mentioned above may be yielded by difference of the damage properties caused by difference of animals, muscles, experimental protocol and so on.

The failure stress for the unstimulated group was obviously lower than for the stimulated group and the control group. Moreover, in multiple regression analyses, the partial regression coefficients of muscle activity and maximum stress on failure stress were positive. These results indicate that muscle activation conduces to higher failure stress. As referred to above, it is reported that the contraction of muscle fibers prevents extensive connective tissue damage (12). In addition, according to electron-microscopic observation of the longitudinal sections after muscle breaking ${ }^{(14)}$, it is hypothesized that there are two load-bearing systems in $\mathrm{Z}$ disks that are capable of force transmission across $Z$ disks: one system consists of passive structural proteins in muscle, such as desmin and titin, and the other system consists of active, thin and thick filaments. From these results and their hypothesis, we infer that a new pathway for 
tension would be formed by muscle contraction and it would hardly work under unstimulated condition. With this hypothesis, most of load to unstimulated muscle would be borne by passive elements of muscle, such as the extracellular connective tissue, cellular membrane, and other structural elements, and as a consequence, damage would be concentrated in the passive elements. Therefore, unlike in the case of the decrease of the contractile force, we consider that the decrease of the failure stress of damaged muscle would reflect the damages of connective tissue and structural element of muscle fiber directly because the failure properties are subject to the mechanical properties of these passive elements.

Additionally, unstimulated muscle would be under influence of stretch reflex even though the animal was anesthetized. Since the aim of this study is to elucidate mechanical properties of skeletal muscle with strain injury, we considered the realistic accident situations. Thus we used the unstimulated condition as the basement line of muscle activation instead of an inactivated condition. For further discussion on the influence of the muscle tone on muscle damage, additional experiments should be done using inactivated skeletal muscle, isolated muscle fascicle, muscle fiber or muscle microstructure.

Our discussion is summarized as follows. While the muscle contractile elements and passive elements are damaged under stimulated condition, the passive elements are damaged severer under unstimulated condition than the contractile elements. In the results, although internal damage under stimulated condition is different from that under unstimulated condition, the decreases of contractile force under both conditions are comparable. The failure stress, which reflects the mechanical properties and damage of passive elements, of the unstimulated group, on the other hand, is lower than that of the stimulated group.

\section{Conclusion}

In this study, we evaluate the effects of biomechanical factors during injury-induction process on the contractile properties and failure properties of skeletal muscle experimentally. It was found that the decrease of contractile force was affected by the stretch amplitude during injury-induction process and was independent of the muscle activity. However, with considering the literature, it is speculated that mechanism of decrease of the contractile force varies from stimulated to unstimulated muscle. While the failure stretch ratio of injured muscle is not different from that of undamaged muscle, the failure stress decreased with the increasing stretch amplitude and dissipation energy. Furthermore, the failure stress of the muscle injured under unstimulated condition was lower than those of the muscle injured under stimulated condition and of undamaged muscle. We conclude that the decrease of the failure stress of damaged muscle would reflect the damages of connective tissue and other structural elements.

\section{Acknowledgement}

We thank Mr. Tsuyoshi Taniguchi, Tatsuya Namikiri, Yoichi Furuyama, Iku Nishizawa, Yuji Tokoro, Arata Tojo, and Yuichiro Watanabe for their great efforts for animal experiments. This work was partially supported by Grant-in-Aid for Scientific Research on Priority Areas 15086208 from the Ministry of Education, Culture, Sports, Science and Technology of Japan.

\section{References}

( 1 ) S. Ejima, K. Ono, S. Holcombe, K. Kaneoka, M. Fukushima, A Study on Occupant Kinematics Behaviour and Muscle Activities during Pre-Impact Braking Based on Volunteer Tests, IRCOBI Conference 2007, 31-45

( 2 ) T. Sugiyama, H. Kimpara, M. Iwamoto, D. Yamada, Y. Nakahira, M. Hada, Effects of muscle tense on impact responses of lower extremity, IRCOBI Conference 2007, 127140

( 3 ) S. Ejima, Y. Zama, F. Satou, S. Holcombe, K. Ono, K. Kaneoka, I. Shiina, Prediction of the Physical Motion of the Human Body based on Muscle Activity during Pre-Impact Braking, IRCOBI Conference 2008, 163-175 
( 4 ) B. J. Gabbe, C. F. Finch, H. Wajswelner, K. Bennell, Australian football: injury profile at the community-level, Journal of Science and Medicine in Sport 5 (2002) 149-160

( 5 ) G. J. Delgado, C. B. Chung, N. Lektrakul, P. Azocar, M. J. Botte, D. C. Coria, Tennis leg: Clinical us study of 141 patients and anatomic investigation of four cadavers with mr imaging and us, Radiology 224 (2002) 112-119.

( 6 ) A. N. Vasavada, J. R. Brault, P. Siegmund, Musculotendon and fascicle strains in anterior and posterior neck muscles during whiplash injury, Spine 32 (2007) 756-765.

( 7 ) P. K. Nikolaou, B. L. MacDonald, R. R. Glisson, A. V. Seaber, W. E. Garrett Jr., Biomechanical and histological evaluation of muscle after controlled strain injury, The American Journal of Sports Medicine 15 (1987) 9-14.

( 8 ) T. J. Noonan, T. M. Best, A. V. Seaber, W. E. Garrett Jr., Identification of a threshold for skeletal muscle injury, The American Journal of Sports Medicine 22 (1994) 257-261.

( 9 ) S. V. Brooks, E. Zerba, J. A. Faulkner, Injury to muscle fibres after single stretches of passive and maximally stimulated muscle in mice, The Journal of Physiology 488 (1995) 459-469.

(10) C. T. Hasselman, T. M. Best, A. V. Seaber, W. E. Garrett Jr., A threshold and continuum of injury during active stretch of rabbit skeletal muscle, The American Journal of Sports Medicine 23 (1995) 65-73.

(11) S. V. Brooks, J. A. Faulkner, Severity of contraction-induced injury is affected by velocity only during stretches of large strain, Journal of Applied Physiology 91 (2001) 661-666.

(12) Y. Uchiyama, T. Tamaki, H. Fukuda, Relationship between functional deficit and severity of experimental fast-strain injury of rat skeletal muscle, European Journal of Applied Physiology 85 (2001) 1-9.

(13) H. Song, K. Nakazato, H. Nakajima, Effect of increased excursion of the ankle on the severity of acute eccentric contraction-induced strain injury in the gastrocnemius: An in vivo rat study, The American Journal of Sports Medicine 32 (2004) 1263-1269.

(14) J. G. Tidball, G. S. Salem, R. Zernicke, Site and mechanical conditions for failure of skeletal muscle in experimental strain injuries, Journal of Applied Physiology 74 (1993) 1280-1286.

(15) Y. S. Hang, Y. H. Tsuang, J. S. Sun, C.K. Cheng, T. K. Liu, Failure of stimulated skeletal muscle mainly contributed by passive force: an in vivo rabbit model, Clinical Biomechanics 11 (1996) 343-347.

(16) T. Taniguchi, S. Yamamoto, A. Hayakawa, E. Tanaka, H. Kimpara, K. Miki, Strain-rate and muscle-tonus dependence of mechanical properties of rabbit tibialis anterior muscle. Proceedings of ATEM : Asian Conference on Experimental Mechanics, OS07W0228 (2003).

(17) T. M. DeBerardino, K. T. Lonergan, D. E. Brooks, Comparison of the split stacked versus the split achilles allograft for dual femoral tunnel posterior cruciate ligament reconstruction, Americal Journal of Sports Medicine 36 (2008) 142-148

(18) L. B. Walker, E. H. Harris, J. V. Benedict, Stress-strain relationship in human cadaveric plantaris tendon: A preliminary study, Medical electronics and Biological Engineering 2 (1964) 31-38

(19) D. C. Taylor, J. D. Dalton Jr., A. V. Seaber, W. E. Garrett Jr., Experimental muscle strain injury. Early functional and structural deficits and the increased risk for reinjury, The American Journal of Sports Medicine 21 (1993) 190-194.

(20) D. M. Scott, A. V. Seaber, R. R. Glisson, W. E. Garrett Jr., The Role of Fatigue in Susceptibility to Acute Muscle Strain Injury, The American Journal of Sports Medicine 24 (1996) 137-143.

(21) R Development Core Team, R: A Language and Environment for Statistical Computing, R Foundation for Statistical Computing, Vienna, Austria, (2008).

URL http: //www.R-project.org 
(22) R. L. Lieber, T. M. Woodburn, J. Fridén, Muscle damage induced by eccentric contractions of $25 \%$ strain, Journal of Applied Physiology 70 (1991) 2498-2507.

(23) J. A. Talbot, D. L. Morgan, The effects of stretch parameters on eccentric exerciseinduced damage to toad skeletal muscle, Journal of Muscle Research and Cell Motility 19 (1998) 237-245.

(24) D. L. Morgan, New insights into the behaviour of muscle during active lengthening, Biophysical Journal 57 (1990) 209-221.

(25) C. L. Brockett, D. L. Morgan, J. E. Gregory, U. Proske, Damage to different motor units from active lengthening of the medial gastrocnemius muscle of the cat, Journal of Applied Physiology 92 (2002) 1104-1110.

(26) J. E. Gregory, D. L. Morgan, T.J. Allen, U. Proske, The shift in muscle's length-tension relation after exercise attributed to increased series compliance, European Journal of Applied Physiology 99 (2007) 431-441. 